REVISTA X, Curitiba, volume 14, n.2, p.199-217, 2019.

\title{
A CONTRIBUIÇÃo DA TRADUÇÃo PEDAGÓGICA NA CORREÇÃo MORFOSSINTÁTICA: UM ESTUDO PRELIMINAR COM ALUNOS DE ESPANHOL COMO LÍNGUA ESTRANGEIRA EM NÍVEL AVANÇADO ${ }^{1}$
}

\begin{abstract}
The Effect of Pedagogic Translation on Morphosyntactic Accuracy: A Preliminary Study with Advanced SSL Students.
\end{abstract}

\author{
Laura GASCA JIMÉNEZ, Universidade de Houston \\ Tradução de Nylcéa Thereza DE SIQUEIRA PEDRA - UFPR
}

\begin{abstract}
RESUMO: No presente artigo são apresentados os resultados de um estudo longitudinal de quatro semanas de duração sobre a contribuição da tradução pedagógica na correção morfossintática da segunda língua. Seis estudantes universitários matriculados em um curso de nível avançado de espanhol como segunda língua na Universidade de Houston participaram do estudo. O curso tinha como objetivo contribuir com o desenvolvimento da competência comunicativa dos estudantes mediante a introdução da prática da tradução direta (L2>L1) e inversa (L1>L2), com ênfase nesta. Os participantes desenvolveram uma atividade de tradução como pré-teste e pós-teste, no começo e no final do curso. Os resultados sugerem que o uso da tradução pedagógica como metodologia ativa que promove a reflexão e combina o trabalho individual com o colaborativo tem um impacto favorável na correção morfossintática da segunda língua. Esse impacto positivo se faz particularmente notório no uso das preposições, dos adjetivos e dos tempos e modos verbais. O presente trabalho se soma aos trabalhos de Cook (2010) e González Davies (2002) que propõem a prática da tradução inversa (L1>L2) para o desenvolvimento da correção gramatical.
\end{abstract}

PALAVRAS-CHAVE: tradução pedagógica; correção morfossintática; tradução inversa

ABSTRACT: This article presents the results of a longitudinal four-week study about the effect of pedagogic translation on morphosyntactic accuracy in a second language. Six undergraduate students enrolled in an advanced Spanish as a second language course at the University of Houston participated in the study. The main objective of this course was to continue developing the students' communicative competence through the introduction of direct (L2>L1) and reverse (L1>L2) translation activities, with an emphasis on the latter. The participants completed a pre and post-test translation activity at the beginning and at the end of the course. The results suggest that the incorporation of pedagogic translation within an active methodology that promotes reflection and combines individual and collaborative work has a significant impact on morphosyntactic accuracy in the target language. This positive impact is particularly noticeable in the use of prepositions, adjectives, and verbal tense and mood. This study contributes to the work of Cook (2010) and González Davies (2002), who propose the practice of reverse translation (L1>L2) for the development of grammatical accuracy. KEYWORDS: pedagogic translation; morphosyntactic; accuracy, reverse translation

\footnotetext{
${ }^{1}$ Artigo publicado originalmente em língua espanhola na Revista Comunicación, vol.12, no.02, 2017. ISSN: 0379-3974. Disponível em: <<http://revistas.tec.ac.cr/index.php/comunicacion/issue/view/408>>
} 
REVISTA X, Curitiba, volume 14, n.2, p.199-217, 2019.

\section{INTRODUÇÃO}

A tradução com fins didáticos vem cada vez mais sendo aceita como uma atividade comunicativa com o potencial de facilitar o ensino e a aquisição de línguas. Os argumentos teóricos que justificam o seu uso são numerosos (CARRERES e NORIEGA-SÁNCHEZ, 2011; COOK, 2010; GONZÁLEZ DAVIES, 2004; COLINA, 2002; MALMKJAER, 1998 e DUFF, 1989), mas os estudos empíricos que avaliam seu potencial como ferramenta didática são poucos (KÄLLKVIST, 2004, 2008 e SÁNCHEZ CUADRADO, 2016).

O presente estudo faz parte de um projeto de pesquisa mais amplo, que tem por objetivo pesquisar o potencial didático da tradução pedagógica no ensino de línguas. Em um estudo prévio, analisou-se o impacto geral da tradução pedagógica no desenvolvimento das habilidades linguísticas e extralinguísticas em estudantes de espanhol como língua estrangeira em nível avançado. Os resultados dessa pesquisa destacam que a incorporação da tradução pedagógica como metodologia ativa e colaborativa que promove a reflexão contribui significativamente para o desenvolvimento das habilidades linguísticas na segunda língua, particularmente da habilidade de transferir informação em atividades de tradução inversa, da primeira para a segunda língua (L1>L2) e da habilidade de usar técnicas de tradução com maiores níveis de correção. Os resultados também indicam que o uso da tradução permite que os estudantes a entendam como uma atividade comunicativa definida por fatores tanto linguísticos quanto extralinguísticos e desenvolvam as habilidades de identificar e respeitar as características que diferenciam as duas línguas.

O presente trabalho é uma ampliação do estudo anteriormente descrito e tem como objetivo investigar a contribuição da tradução no aperfeiçoamento das habilidades morfossintáticas na segunda língua. Os resultados sugerem que a prática da tradução inversa (L1>L2) contribui positivamente para a correção morfossintática. Apesar de ser um estudo de caráter preliminar, por contar com um número reduzido de participantes e basear-se na análise de um número limitado de amostras de escrita, a presente pesquisa é de suma relevância para o campo da tradução pedagógica, uma vez que valida empiricamente seu valor didático no ensino de segundas línguas.

O artigo está dividido em seis partes: na primeira, são apresentados os principais referenciais teóricos relacionados à tradução pedagógica; na segunda, são incluídos os 
REVISTA X, Curitiba, volume 14, n.2, p.199-217, 2019.

estudos empíricos feitos anteriormente nesta mesma linha de pesquisa; na terceira, explica-se a metodologia empregada; na quarta, são descritos e analisados os resultados obtidos; na quinta, apresentam-se as conclusões e implicações pedagógicas são propostas a partir delas e, por último, são apresentadas as limitações do estudo.

\title{
REFERENCIAL TEÓRICO
}

Desde o já abandonado método gramática-tradução, a tradução passou a ser ignorada como possível ferramenta didática. Como afirma o reconhecido linguista Guy Cook (2010), a ideia de que a tradução não é benéfica para a aquisição de línguas é tão generalizada que sequer existem muitos estudos sobre o assunto. Além de ter sido relegada ao exílio, também foi desprezada por ser considerada uma atividade extremamente complexa e trabalhosa, à margem das quatro habilidades que conformam a competência linguística. Ela, além de atrapalhar a aprendizagem de uma segunda língua (doravante, L2), promove a transferência negativa ${ }^{2}$ da língua materna (doravante, L1). Alan Maley expressa tudo isso de maneira clara no prólogo do livro intitulado Translation do linguista, professor e tradutor Alan Duff, publicado em 1989:

\begin{abstract}
A tradução definhava há bastante tempo no ensino de línguas. Foi denegrida com comentários como "não comunicativa", "chata", "sem sentido", "difícil", "irrelevante" e sofreu com a aproximação que fizeram entre ela e a gramática. Junto com outros métodos mais tradicionais como a literatura, o ditado, o vocabulário e a leitura em voz alta, foi vista como um método arcaico de ensino (p.3)
\end{abstract}

Muitos autores coincidem que a reputação negativa da tradução se deve ao método gramática-tradução (MALMKJAER, 1998; GONZÁLEZ, 2004 e COOK, 2010, entre outros). O objetivo desse método era facilitar a leitura de literatura na língua meta, motivo pelo qual só eram trabalhadas a compreensão leitora e a produção escrita. As regras gramaticais eram apresentadas e memorizadas para serem utilizadas na tradução de orações de ou para a língua meta ${ }^{3}$. Com a chegada do método direto e a consequente ênfase na língua falada, a tradução foi expulsa das aulas de língua para dar lugar ao que Cummins (2008) denomina o princípio do monolinguismo: a primeira e a segunda língua se mantêm separadas uma da outra durante a aprendizagem.

\footnotetext{
2 Ao longo do presente trabalho a transferência negativa será entendia como o desvio das normas linguísticas monolíngues por influência de outra língua.

${ }^{3}$ Para explicação mais detalhada consultar Richards e Rodgers (2014).
} 
REVISTA X, Curitiba, volume 14, n.2, p.199-217, 2019.

Os argumentos mais frequentes aos quais recorreram vários autores - como Lado (1964) e Gatenby (1967) - para negar a utilização da tradução são os de que não se trata de um processo natural, que cria uma falsa ilusão de equivalência entre as duas línguas e promove a transferência negativa da L1. Lado (1964) defende que a tradução não permite a prática da L2 uma vez que cria a falsa ilusão de equivalência que compromete a correção gramatical e favorece as traduções literais agramaticais. Além disso, afirma que a tradução é um processo complexo e desnecessário para desenvolver a expressão oral, a expressão escrita, a compreensão auditiva e a compreensão leitora. Em resumo, o autor entende a tradução como uma atividade contraproducente, ao menos que se domine uma segunda língua: "Uma boa tradução não pode ser alcançada sem o domínio de uma segunda língua. Por isso é preciso ensinar a língua primeiro para, então, se for considerado desejável, ensinar a tradução como uma habilidade separada" (LADO, 1964, p.54). Para Gatenby (1967), a tradução é uma atividade artificial e contraproducente que deveria ser evitada a todo custo como atividade e como ferramenta de avaliação. Como Lado (1964), descarta o potencial didático da tradução, relegando-a ao status de habilidade que depende do domínio de duas línguas:

\begin{abstract}
A tradução é um processo enganoso. Sendo trabalhosa, convence professor e aluno que fizeram muita coisa. Infelizmente, esse trabalho é inútil. Tradução pode dar significado, mas não ensina. Perpetua o hábito de perder tempo associando a nova língua com a conhecida, dificultando a compreensão total. Como habilidade, o momento adequado para praticá-la é quando se domina igualmente ambas línguas. (GATENBY, 1967, p.70)
\end{abstract}

Nas últimas décadas, foram apresentados inúmeros argumentos para reivindicar o lugar da tradução com fins didáticos dentro do método de ensino comunicativo e das metodologias ativas. Cook (2010) e González Davies (2002) enfatizam o desenvolvimento da correção gramatical, particularmente mediante a prática da tradução inversa (L1>L2). Segundo os autores, a prática da tradução, diferentemente da escrita livre, obriga os alunos a enfrentar as dificuldades da segunda língua:

Vamos pensar sobre o que acontece quando os alunos escrevem uma redação, eles podem evitar áreas problemáticas e se comunicar de forma eficaz. Com a tradução, precisam retransmitir o que está no texto de origem, eles precisam ter um controle linguístico e extralinguístico para que isso aconteça. (GONZÁLEZ DAVIES, 2002, p.70) 
REVISTA X, Curitiba, volume 14, n.2, p.199-217, 2019.

Cook (2010) reconhece a importância de se desenvolver a habilidade de parafrasear mediante atividades abertas, mas enfatiza a utilidade de se combinar essas atividades com exercícios de tradução que permitam se centrar nas dificuldades específicas do processo de aprendizagem:

\begin{abstract}
A tradução não precisa ser vista apenas como um elemento dificultador, ela pode ser usada proativamente a fim de chamar a atenção dos alunos para aspectos difíceis da nova língua. Para o professor ou para o escritor de materiais que já conhece a língua dos alunos e as dificuldades que podem apresentar durante o aprendizado da nova língua, é possível pensar em atividades de tradução que concentrem a atenção nessas áreas problemáticas (COOK, 2010, p.138)
\end{abstract}

Vários autores enfatizam que a tradução permite controlar a transferência negativa e fomentar a transferência positiva. Snell-Hornby (1985), baseando-se em sua experiência como professora de uma disciplina de tradução alemão-inglês, se opõe ao rechaço absoluto da tradução em todos os níveis de aquisição de uma segunda língua. A autora sugere que a prática da tradução permite explorar as relações interlinguísticas e contribui positivamente para a correção gramatical. Na mesma linha, Titford (1985) argumenta que as atividades de tradução são o contexto idôneo para explicar a transferência negativa da L1 sem a necessidade de se recorrer a explicações de cunho metalinguístico. Sorensen (1990) enfatiza o valor didático de comparar e contrastar as duas línguas mediante a prática direta e inversa da tradução, particularmente no caso de alunos de nível avançado: "esta atividade torna os estudantes de nível avançado mais conscientes das divergências e incongruências entre as duas línguas” (p.202). Harvey (1996) destaca a utilidade da tradução para tornar evidentes os erros que podem ser o resultado de uma superposição inconsciente das estruturas da língua materna. Malmkjaer (1998) reitera que a tradução contribui para o desenvolvimento da habilidade de relacionar dois sistemas linguísticos, o que permite minimizar a interferência negativa e maximizar a positiva. Também afirma que é inegável que a tradução cria interferência, mas defende que a prática da tradução a coloca em evidência e permite o desenvolvimento de mecanismos de controle para limitá-la.

Assim, apesar dos pré-conceitos existentes sobre a tradução como consequência da sua relação com o método gramática-tradução, numerosos autores destacam que se trata de uma atividade comunicativa com o potencial de contribuir para o aperfeiçoamento da correção gramatical e para a diminuição da transferência negativa. 
REVISTA X, Curitiba, volume 14, n.2, p.199-217, 2019.

\section{ESTUDOS PRELIMINARES}

É importante enfatizar que a tradução pedagógica é um campo de pesquisa aberto, dado que são poucas as pesquisas empíricas realizadas até o momento para validar sua aplicação (CARRERES e NORIEGA-SÁNCHEZ, 2011; CUMMINS, 2007; HURTADO ALBIR, 2001 e KÄLLKVIST, 2008). Foram desenvolvidos vários estudos empíricos que pesquisam seu potencial como ferramenta de avaliação (KOBAYASHI E RINNERT, 1992; KÄLLKVIST, 1996; COHEN e BROOKS-CARSON, 2001 e SCHJOLDAGER, 2004), no entanto, poucos são os estudos empíricos que analisaram os efeitos que produz como ferramenta didática (SNELLINGS VAN GELDEREN e DE GLOPPER, 2002; KÄLLKVIST, 2004, 2008; VAEZI e MIRZAEI, 2007; LAUFER e GIRSAI, 2008; BELPOLITI e PLASCENCIA-VELA, 2013 e SÁNCHEZ CUADRADO, 2016). Apresentamos com mais detalhes duas dessas pesquisas que analisam especificamente o impacto da tradução no desenvolvimento das habilidades morfossintáticas.

Källkvist (2004) pesquisa a possibilidade de aperfeiçoamento do uso de estruturas morfossintáticas em uma L2 mediante a prática da tradução. 44 universitários que tem o sueco como língua materna participaram desse estudo de treze semanas de duração. Os participantes foram divididos em três grupos, dois experimentais e um não experimental. O primeiro grupo experimental (o grupo de tradução) realizou de maneira consistente exercícios de tradução para trabalhar estruturas morfossintáticas específicas, enquanto o segundo (o grupo de não tradução), trabalhou com as mesmas estruturas, mas com exercícios na L2. O grupo não experimental estava formado por alunos do último ano que não recebeu instrução explícita de gramática na L2, mas uma grande exposição à língua mediante a leitura e a escrita. Todos os participantes completaram um pré-teste que constava de três tarefas: uma atividade de múltipla escolha, uma atividade de tradução e uma atividade de reescrita. Depois do período de desenvolvimento da pesquisa, todos os participantes voltaram a completar as mesmas tarefas. Além do pré-teste e do pós-teste, todos realizaram uma prova mais curta durante a terceira semana da pesquisa que constava de um exercício de tradução e um de completar lacunas. Essa prova tinha como objetivo avaliar a aquisição de uma estrutura gramatical estudada (o uso do artigo definido).

Os resultados da pesquisa se limitaram à análise da prova. Pelo número limitado de itens (3), os resultados devem ser interpretados como provisórios. Esse estudo mostra 
REVISTA X, Curitiba, volume 14, n.2, p.199-217, 2019.

que, no que cabe ao uso do artigo definido, os estudantes com motivação alta que são expostos a exercícios na L2 podem ter desempenho igual ou superior em um exercício de tradução que os estudantes expostos a esta. Os resultados gerais evidenciam que os dois grupos experimentais obtiveram resultados similares, enquanto o grupo não experimental teve resultados consideravelmente inferiores. A autora recomenda combinar exercícios de tradução com outros na L2 que permitam se centrar na forma.

Källkvist (2008) retoma os dados de seu estudo preliminar de 2004 para comparar de maneira mais detalhada os efeitos do uso dos exercícios de tradução inversa (L1>L2) e dos exercícios na L2 (completar lacunas e de transformação) na aprendizagem de L2. A pesquisa foi realizada durante 12 semanas. A análise dos prétestes e dos pós-testes mostra que não existe uma diferença significativa entre os resultados dos dois grupos nos exercícios de múltipla escolha e tradução. No entanto, a autora destaca que no exercício de tradução, o grupo de tradução obteve resultados que se aproximam ao nível de significância estatística e conjuntura e que, com um grupo maior de participantes e um número maior de itens, as diferenças observadas entre os dois grupos no exercício de tradução teriam sido estatisticamente significativas. Os resultados do estudo sugerem que os estudantes de nível avançado podem beneficiar-se da tradução, não como única forma de explicação, mas em combinação com outros exercícios na L2.

\section{METODOLOGIA}

Dentro da mesma linha de pesquisa que os estudos apresentados na seção anterior, o presente estudo colabora para a pesquisa sobre a contribuição da tradução pedagógica na correção linguística, área que, como já destacamos, carece de mais estudos quantitativos.

\section{Perguntas de pesquisa:}

a) Há um aumento no número de erros morfossintáticos depois do período de realização da pesquisa?

b) Há uma diminuição no número de erros morfossintáticos depois do período de realização da pesquisa?

c) Que categorias morfossintáticas foram afetadas positiva ou negativamente depois do período de realização da pesquisa?

\section{Participantes:}


REVISTA X, Curitiba, volume 14, n.2, p.199-217, 2019.

Participaram oito estudantes de espanhol matriculados no nível avançado para alunos de Licenciatura da Universidade de Houston. A disciplina faz parte da especialização em espanhol a qual os estudantes podem aceder de maneira optativa depois de completar satisfatoriamente as duas disciplinas básicas e as duas intermediárias de espanhol como segunda língua. No momento em que o estudo foi desenvolvido, os estudantes estavam em intercâmbio, em Cádiz, Espanha. Dois estudantes foram descartados por não estarem presentes no momento da apresentação dos instrumentos. Desse modo, o número de participantes ficou reduzido a seis (ver Tabela 1).

Tabela 1: Características dos participantes

\begin{tabular}{ccccc}
\hline Ref. Gênero & $\begin{array}{c}\text { Aulas de } \\
\text { espanhol } \\
\text { (ensino } \\
\text { fundamen- } \\
\text { tal) }\end{array}$ & $\begin{array}{c}\text { Aulas de } \\
\text { espanhol } \\
\text { (ensino } \\
\text { médio) }\end{array}$ & $\begin{array}{c}\text { Aulas de } \\
\text { espanhol } \\
\text { (universi- } \\
\text { dade) }\end{array}$ \\
P1 & M & 2 anos & 3 anos & 3 semestres \\
P3 & F & 1 ano & 4 anos & 4 semestres \\
P4 & F & 3 anos & 1 ano & 2 semestres \\
P5 & F & - & 2 anos & 5 semestres \\
P6 & F & 2 anos & 3 anos & 1 semestre \\
P7 & F & 3 anos & 2 anos & 3 semestres
\end{tabular}

Instrução:

O objetivo geral do curso foi contribuir para o desenvolvimento da competência comunicativa dos estudantes mediante a introdução da prática da tradução direta (do espanhol para o inglês) e da tradução inversa (do inglês para o espanhol), enfatizando esta última. Foi um curso intensivo, com quatro encontros semanais (de segunda a quinta-feira), de duas horas de duração, durante quatro semanas consecutivas. Seguindo as propostas de Kiraly (2000) e González Davies (2004), utilizou-se uma metodologia ativa, centrada no estudante, priorizando o processo (a prática de tradução) e não o produto (o texto traduzido).

Durante os primeiros encontros foram apresentadas diferentes fontes de consulta (dicionários bilíngues, monolíngues, corpora, etc.) que possibilitaram que os alunos encarassem a tradução como uma atividade interpretativa que depende de fatores extralinguísticos como, por exemplo, o objetivo do texto e o público ao qual se dirige. Os outros encontros se centraram na prática colaborativa de tarefas de tradução, como 
REVISTA X, Curitiba, volume 14, n.2, p.199-217, 2019.

também na reflexão em grupo sobre as características dos textos, os problemas de tradução, as possíveis soluções, etc. A partir dessas atividades, foi sendo apresentada a terminologia relacionada à prática profissional de tradução. Foram apresentadas diferentes técnicas de tradução, foram analisadas as principais marcas diferenciais entre o inglês e o espanhol a nível sintático, morfológico e de pontuação e, finalmente, se explorou a dimensão social da tradução e a importância de se considerar as variantes linguísticas do espanhol ao traduzir. Além das atividades colaborativas em sala, a cada semana os alunos tiveram que trabalhar individualmente em uma tradução, que deveria vir acompanhada por uma breve reflexão sobre as características do texto, as principais dificuldades encontradas e as soluções postas em prática (ver Anexo A com exemplo de tarefa e entrega).

\section{Instrumentos}

Foi utilizada uma atividade de tradução inversa (L1>L2) como pré-teste/pósteste para avaliar o impacto do período de treinamento (veja-se Anexo B). O texto de origem da tradução é um fragmento de 160 palavras de uma notícia publicada em inglês na página Yahoo News sobre o lançamento de um novo produto da Apple. Foi escolhido por se tratar de um tema fácil e atual, mas sua terminologia semi-técnica motivava o uso de técnicas de tradução.

\section{Procedimento}

Os participantes realizaram o pré-teste e o pós-teste durante o primeiro e o último dia de aula. Tiveram uma hora para realizá-los e não foi permitido usar material de consulta. Ao longo do curso, não tiveram contato com a atividade e nem foram informados de que voltariam a fazê-la ao final, como recomendam Mellinger e Hanson (2017), para garantir a validade dos resultados em pesquisas que fazem uso desse procedimento.

Partindo da técnica de análise de erros (JAMES, 1998) e outros estudos que realizaram análises similares com estudantes de espanhol (FAIRCLOUGH e MRAK, 2003), foi desenvolvida uma classificação de erros que permitiu quantificar os erros morfossintáticos encontrados nas traduções. Foram estabelecidas nove categorias de erros: concordância dentro da frase nominal $(\mathrm{CN})$, concordância entre o sujeito e o verbo (CV), tempo e modo (TM), preposições (PR), advérbios (ADV), clíticos (CL), 
REVISTA X, Curitiba, volume 14, n.2, p.199-217,2019.

adjetivos (ADJ), ser e estar (SE) e outros (OT). Essas categorias foram subdivididas em cinco tipos de erros: omissão, adição, substituição, ordem e combinação ${ }^{4}$. Os erros encontrados nas traduções foram quantificados e classificados segundo essa classificação. Para calcular o número total de palavras produzidas durante o período de treinamento, foi somado o total de palavras, excluindo os comentários em língua inglesa feitos pelos estudantes para justificar a escolha ou eliminação de expressões.

\section{Resultados e discussão}

O número total de palavras no pré-teste foi de 897 , das quais 125 entendidas como erro $(0,13 \%)$. No pós-teste, o número de palavras aumentou para 936 e o número total de erros diminuiu para $89(0,09 \%)$, como se pode ver na tabela 2 . As categorias linguísticas com maior incidência de erros durante os dois processos de avaliação foram: TM (Pré= 0,30; Pós= 0,28), CN (Pré=0,22; Pós=0,24), PR (Pré=0,22; Pós= 0,16) e ADJ (Pré= 0,12; Pós=0,07). O número de erros nas categorias CV, ADV, CL, SE e OT foi muito pequeno, razão pela qual não são incluídos na análise dos resultados pré-teste/pós-teste.

Tabela 2: Número e frequência de erros morfossintáticos no pré-teste e pós-teste

\begin{tabular}{|c|c|c|c|c|c|c|c|c|c|c|c|c|c|c|c|c|c|c|c|c|}
\hline \multirow[b]{4}{*}{ Omissão } & \multicolumn{16}{|c|}{ Categorias linguísticas } & \multirow{2}{*}{\multicolumn{2}{|c|}{ OT }} & \multirow{2}{*}{\multicolumn{2}{|c|}{ Total }} \\
\hline & \multicolumn{2}{|c|}{$\mathbf{C N}^{\mathrm{a}}$} & \multicolumn{2}{|l|}{$\mathrm{CV}$} & \multicolumn{2}{|c|}{$\begin{array}{c}\mathbf{T} \\
\mathbf{M}\end{array}$} & \multicolumn{2}{|c|}{ PR } & \multicolumn{2}{|c|}{ ADV } & \multicolumn{2}{|c|}{ CL } & \multicolumn{2}{|c|}{ ADJ } & \multicolumn{2}{|c|}{$\mathrm{SE}$} & & & & \\
\hline & Préb $^{\mathrm{b}}$ & Pós & & éPós & Pré & Pós & & réPós & Pré & Pós & & réPós & Pré & Pós & & réPós & Pré & Pós & Pré & Pós \\
\hline & $6^{c}$ & 6 & 1 & - & 7 & 3 & 2 & 3 & 2 & 1 & 2 & 2 & 1 & 1 & - & 1 & 3 & 2 & 24 & 19 \\
\hline Adição & 1 & - & - & - & 13 & 4 & 12 & 8 & - & 1 & 1 & 3 & 2 & 1 & - & - & - & 1 & 30 & 18 \\
\hline Substituição & 21 & 16 & 3 & 3 & 18 & 17 & 12 & 4 & 1 & - & - & - & 4 & 1 & 3 & 3 & - & - & 62 & 44 \\
\hline Ordem & - & - & - & - & - & 1 & - & - & - & - & - & - & 8 & 4 & - & - & - & 1 & 8 & 6 \\
\hline Combinação & - & - & - & - & - & - & 2 & - & - & 2 & - & - & - & - & - & - & - & - & 2 & 2 \\
\hline Total & 28 & 22 & 4 & 3 & 38 & 25 & 28 & 15 & 3 & 4 & $J$ & 5 & 15 & 7 & 3 & 4 & 3 & 4 & 125 & 89 \\
\hline $\operatorname{Total}^{\mathrm{d}}(\%)$ & 0,22 & 0,24 & 0,03 & 0,03 & 0,30 & 0,28 & 0,22 & 0,16 & 0,02 & 0,04 & 0,02 & 0,06 & 0,12 & 0,07 & 0,02 & 0,04 & 0,02 & 0,04 & 0,13 & 0,09 \\
\hline
\end{tabular}

${ }^{\mathrm{a}} \mathrm{CN}=$ concordância nominal, $\mathrm{CV}=$ concordância verbal, $\mathrm{TM}=$ tempo e modo, $\mathrm{PR}=$ preposições, $\mathrm{ADV}=$ advérbios, $\mathrm{CL}=$ clíticos, $\mathrm{ADJ}=$ adjetivos, $\mathrm{SE}=$ ser e estar, $\mathrm{OT}=$ outros

${ }^{\mathrm{b}}$ Pré-teste e pós-teste

${ }^{\mathrm{c}}$ Número de casos

${ }^{\mathrm{d}}$ Frequência de erros

A categoria TM inclui os seguintes tipos de erros: substituição de um tempo verbal por outro (exemplo 1) ou de um modo por outro (exemplo 2), a adição de formas novas (exemplo 3) e a omissão de verbos (exemplo 4):

\footnotetext{
${ }^{4}$ Refere-se à combinação de duas formas que tem como resultado uma combinação inexistente na língua em questão.
} 
REVISTA X, Curitiba, volume 14, n.2, p.199-217, 2019.

1. No es completamente de sorpresa que Apple querria traer esa option de Pro a Air standard. (P1/Pré-teste)

2. (...) pero sería sorprendente de ver que pasa después de unos pocos meses. (P4/Pré-teste)

3. Apple probablemente introducionar un modelo nuevo del Air. (P6/Préteste)

4. La más importante es que el cito dice que Apple ha [X] un modelo del Air que trabaja con el Apple Pencil. (P6/Pré-teste5)

Nessa categoria, o tipo de erro que apresenta maior melhora na comparação entre o pré-teste e o pós-teste é a adição de formas novas, isto é, a ocorrência de um número menor de criações léxicas no pós-teste. A omissão também diminuiu, mas a substituição de formas se manteve praticamente igual. Os próximos exemplos permitem observar a evolução individual de cinco dos seis participantes do estudo que no pré-teste recorreram a criações léxicas ${ }^{6}$ e que no pós-teste foram capazes de empregar formas existentes em espanhol:

5. Apple probablemente introducionar un modelo nuevo del Air. (P6/Préteste)

6. Apple probablemente va a introducir un modelo con mejoras del Air. (P6/Pós-teste)

7. No hay mucho detales a que cuando vamos a expecto para el nuevo IPad moda. (P7/Pré-teste)

8. No hay muchos detalles sobre a que el nuevo iPad model tiene. (P7/Pósteste)

9. El sitio llame más atención en lo que Apple está expirimentando una versión del Air que funciona con el Apple Pencil. (P5/Pré-teste)

10. Más importante, dice el sitio que Apple está probando una versión del Air que funciona con el Apple Pencil. (P5/Pós-teste)

11. (...) cuando lo anunica el iPad pro y iPad mini 4. (P3/Pré-teste)

12. (...) cuando lo anunció el iPad Pro y iPad mini 4. (P3/Pós-teste)

13. (...) pero el cita de "9to5Mac" nos directa a los ideas mas temepranos. (P1/Pré-teste)

14. (...) pero la cita de "9to5Mac" pone atención a las rumores. (P1/Pré-teste)

A categoria CN conta com casos de substituição de gênero (exemplo 15) ou número (exemplo 16), omissão de artigos determinados (exemplo 17) e um caso de adição de artigo determinado (exemplo 18):

15. No hay muchas detallas abiertas sobre en el modelo de iPad nuevo. (P1/Pré-teste)

\footnotetext{
${ }^{5}$ 1) quisiera; 2) pasaría; 3) introducirá; 4) presentado/desarrollado.

${ }^{6}$ As criações léxicas implicam em uma transferência de forma e significado e se diferenciam dos empréstimos na frequência de uso e nível de integração social. Enquanto os empréstimos costumam ser utilizados nas comunidades de fala, as criações léxicas costumam pertencer aos idioletos individuais dos falantes (MUÑOZ SÁNCHEZ, 2007).
} 
REVISTA X, Curitiba, volume 14, n.2, p.199-217, 2019.

16. (...) dicen que el Air nuevo puede recibe adicional hablantes y un flash para la camera. (P3/Pré-teste)

17.(...) que la nueva air podra tener bocinas y $[\underline{X}]$ flash adicional para su camara. (P4/Pré-teste)

18. (...) pero el cita de "9to5Mac" nos directa a los ideas mas temepranos. (P1/Pré-teste $)^{7}$

Nessa categoria, o único tipo de erro que apresenta uma pequena melhora é a substituição, o que parece sugerir que os participantes têm um maior controle das categorias de gênero e número. $\mathrm{O}$ caso de adição desaparece no pós-teste e o número de omissões se mantêm igual. Os próximos exemplos ilustram a evolução individual dos participantes:

19. Notablemente, la sita dice que Apple ha sido examinar un versión de Air que trabaja con el lápiz Apple. (P3/Pré-teste)

20. Notable más, el sitio dice que Apple ha sido examinando una versión de el Air que trabajó junto con el Apple Lápiz. (P3/Pós-teste)

21. (...) pero este fall pasada, ha escogido que no aparece el Air 3. (P5/Préteste)

22. (...) pero no sube un Air 3 este otoño pasado. (P5/Pós-teste)

23. No hay mucho informacion sobre expectaciones del iPad modelo nuevo. (P6/Pré-teste)

24. No hay muchos detalles sobre las expectaciones del nuevo iPad modelo. (P6/Pós-teste)

É importante destacar que, a pesar de o número de substituições ter diminuído ligeiramente no pós-teste, a concordância de gênero continuou problemática, o que se reflete na presença de erros desse tipo durante o segundo período de avaliação:

25. (...) mostrando la primera cambia de la linea en mas que un año. (P1/Pósteste)

26. No hay muchas detalles en lo que expectativas buscar de el nuevo modelo de iPad. (P3/ Pós-teste)

27. (...) que el Air nuevo va a tener sonadores adicionales y una luz para el camera detrás. (P5/Pós-teste)

28. El sitio dice que Apple estaba examinando un version de el Air que trabaja con el Apple Pencil. (P7/Pós-teste)

Na categoria PR, os principais exemplos são de substituição de uma preposição por outra e adição de preposições desnecessárias (exemplos 29 a 31). A frequência de substituições melhorou consideravelmente durante a segunda avaliação, como pode ser visto nos exemplos 32 e 33 :

\footnotetext{
${ }^{7}$ 15) detalles; 16) hablantes adicionales; 17) un flash; 18)_a ideas.
} 
REVISTA X, Curitiba, volume 14, n.2, p.199-217, 2019.

29. No hay muchas detallas abiertas sobre en el modelo de iPad nuevo. (P1/Pré-teste)

30. El sitio llame más atención en lo que Apple está expirimentando una versión del Air que funciona con el Apple Pencil. (P5/Pré-teste)

31. (...) pero Apple no inducir a el Air 3 en el fall pasado. (P7/Pré-teste) ${ }^{8}$

32. (...) que muestra el primer cambio en mas que un año dela linea. (P6/Préteste)

33. (...) mostrando el primer cambio de la linea en mas de un ano. (P6/Pósteste)

$\mathrm{Na}$ categoria ADJ, foram identificados principalmente casos de ordem, nos quais a posição do adjetivo foi alterada, e de substituição, nos quais o adjetivo foi substituído por um substantivo. Os casos de substituição praticamente desapareceram no pós-teste, enquanto os de ordem diminuíram, mas continuaram presentes:

34. No hay muchas detalles en como que expectativa de el iPad nuevo modelo. (P3/Pré-teste)

35. No hay muchas detalles en lo que expectativas buscar de el nuevo modelo de iPad. (P3/ Pós-teste)

36. pero el "9to5Mac" cito refleja chisme. (P6/ Pré-teste)

37. pero el sito de "9to5Mac" se implica a los rumores tempranos. (P6/Pósteste)

38. No es una sorpresa que Apple quiere esta misma función del Pro al Air estándar. (P5/ Pré-teste)

39. No es sorprendente que Apple quiere este funciona en el Air estándar igual que el Pro. (P5/Pós-teste)

Em resumo, a pesar do pouco tempo transcorrido entre o pré-teste e o pós-teste, a análise dos dados demonstra uma ligeira diminuição dos erros morfossintáticos depois do período de realização da pesquisa, o que sugere que a incorporação da tradução pedagógica tem um impacto positivo no desenvolvimento da precisão morfossintática na segunda língua. $\mathrm{O}$ aumento da precisão morfossintática é particularmente notável no uso das preposições, adjetivos e tempos e modos verbais. Depois do período de realização da pesquisa, os participantes apresentaram maior domínio ao selecionar as preposições adequadas em contextos variados, assim como a tendência de incluir com menos frequência preposições desnecessárias. No que cabe aos adjetivos, tiveram menos dificuldades para posicioná-los em relação ao substantivo que modificam e para manter a categoria linguística que assumiam no texto de origem também no texto meta, isto é, para traduzir os adjetivos como adjetivos sem substituí-los por substantivos. Por último, no que cabe aos tempos e modo verbais, demonstraram uma maior habilidade

\footnotetext{
8 29) Sobre [x] el modelo; 30) atención de; 31) [x] el otoño pasado.
} 
REVISTA X, Curitiba, volume 14, n.2, p.199-217, 2019.

para usar as formas verbais existentes na língua sem ter que recorrer a uma criação léxica.

A presença de erros morfossintáticos no pré-teste não deveria surpreender, uma vez que - como já apontaram outros autores - na tradução, ao contrário da escrita livre, os estudantes não podem recorrer ao que González Davies (2002) denomina estratégias de evasão para evitar os aspectos linguísticos que lhes resultam problemáticos ou que lhes causam insegurança.

\section{CONCLUSÕES E IMPLICAÇÕES PEDAGÓGICAS}

A menor frequência de erros morfossintáticos depois do período de realização da pesquisa sugere que a incorporação da tradução pedagógica nas aulas de nível avançado mediante uma metodologia ativa que combina o trabalho individual e o colaborativo e promove a reflexão tem um impacto considerável na correção morfossintática na segunda língua. Esse impacto positivo é particularmente notável no uso de preposições, adjetivos e tempos e modos verbais.

Os resultados deste estudo contradizem as afirmações de Lado (1964) e Gatenby (1967) sobre as consequências negativas da prática da tradução na correção gramatical e se une a trabalhos de autores como Cook (2010) e González Davies (2002), que propõem a prática da tradução inversa (L1>L2) para desenvolver a correção gramatical.

Finalmente, como destacaram outros pesquisadores (CARRERES e NORIEGASÁNCHEZ, 2011; CUMMINS, 2007; HURTADO ALBIR, 2001 e KÄLLKVIST, 2008) é conveniente destacar a importância de se realizar mais pesquisas empíricas que avaliem os efeitos da tradução pedagógica.

\section{LIMITAÇÕES}

Apesar de o estudo preliminar oferecer resultados muito interessantes, apresenta algumas limitações: (i) o número de participantes foi limitado, uma vez que dependia do número de estudantes matriculados no curso; (ii) não foi incluído um grupo de controle; (iii) não foi possível realizar um segundo pós-teste, uma vez que a maioria dos estudantes se graduou e deixou a instituição.

\section{REFERÊNCIAS}


REVISTA X, Curitiba, volume 14, n.2, p.199-217, 2019.

BELPOLITI, Flavia PLASCENCIA-VELA, Amira. Translation techniques in the spanish for heritage learners' classroom: Promoting lexical development. In: Translation in language teaching and assessment. Newcastle: Cambridge Scholars Publishing, 2013. p. 65-91.

CARRERES, Ángeles e NORIEGA-SÁNCHEZ, María. Translation in language teaching: Insights from professional translator training. The Language Learning Journal. Vol. 39, 2011, p. 281-297.

COHEN, Andrew e BROOKS-CARSON, Amanda. Research on direct versus translated writing: Students' strategies and their results. The Modern Language Journal, no.85, 2001, p. 169-188.

COLINA, Sonia. Second language acquisition, language teaching and translation studies. The Translator, vol. 8, 2002, p. 1-24.

COOK, Guy. Translation in language teaching: An argument for reassessment. Oxford: Oxford University Press, 2010.

CUMMINS, Jim. Rethinking monolingual instructional strategies in multilingual classrooms. Canadian Journal of Applied Linguistics/Revue canadienne de linguistique appliquée, no.10, 2007, p. 221-240.

Teaching for transfer: Challenging the two solitudes assumption in bilingual education. Encyclopedia of language and education. New York: Springer Science, 2008, p. 65-77.

DUFF, Alan. Translation. Oxford: Oxford University Press, 1989.

FAIRCLOUGH, Marta e MRAK, Ariana. La enseñanza del español a los hispanohablantes bilingües y su efecto en la producción oral. Mi lengua: Spanish as a heritage in the United States, research and practice. Washington D.C.: Georgetown UP, 2003, p. 198-212.

GASCA JIMÉNEZ, Laura. Las posibilidades de la traducción pedagógica para la enseñanza del español como segunda lengua. Estudios de Lingüística Aplicada, 66. (no prelo)

GATENBY, Eve. Popular fallacies in the teaching of foreign languages. ELT Journal, no. 7, 1967, p. 21-29.

González Davies, María. Translation in foreign language learning: Sleeping with the enemy? APAC of news, 2002, p.64-74.

Multiple voices in the translation classroom: Activities, tasks and projects. Amsterdam: J. Benjamins, 2004.

HARVEY, Malcon. A translation course for french-speaking students. Teaching translation in universities: Present and future perspectives Londres: Middlesex University Printing Services, 1996, p. 15-30. 
REVISTA X, Curitiba, volume 14, n.2, p.199-217, 2019.

Hurtado Albir, Amparo. Traducción y traductología: Introducción a la traductología. Madrid: Cátedra, 2001.

James, Carl. Errors in language learning and use: Exploring error analysis. New York: Routledge, 1998.

KÄLLKVIST, Marie. How different are the results of translation tasks? A study of lexical errors. Translation and language teaching. Manchester: St. Jerome Publishing, 1996, p. 77-89.

The effect of translation exercises versus gap-exercises on the learning of difficult 12 structures. Translation in undergraduate degree programmes, vol. 59, 2004, p. 163-184.

L1-12 translation versus no translation: A longitudinal study of focus-on-forms within a meaning-focused curriculum. The longitudinal study of advanced 12 capacities. New York: Routledge, 2008, p. 182-202.

KIRALY, Don. A social constructivist approach to translator education: Empowerment from theory to practice. Manchester: St. Jerome Pub, 2000.

KOBAYASHI, Hiroe. e RINNERT, Carol. Effects of first language on second language writing: Translation versus direct composition. Language Learning, no. 42, 1992, p. 183-209.

LADO, Robert. Language teaching. A scientific approach. New York: McGraw-Hill, 1964.

LAUFER, Batia e GIRSAI, Nany. Form-focused instruction in second language vocabulary learning: A case for contrastive analysis and translation. Applied Linguistics, no. 29, 2008, p. 694-716.

MALMKJÆR, Kirsten. Translation and language teaching: Language teaching and translation. Manchester: St. Jerome Publishing, 1998.

MELLINGER, Christopher e HANSON, Thomas. Quantitative research methods in translation and interpreting studies. New York: Routledge, 2007.

MUÑOZ SÁNCHEZ, Ama. Register and style variation in speakers of spanish as a heritage and as a second language. Tese de doutorado, 2007. Disponível em: << http://digitallibrary.usc.edu/cdm/ref/collection/p15799coll127/id/327976>>

RICHARDS, Jack e RODGERS, Theodore. Approaches and Methods in Language Teaching. UK: Cambridge University Press, 2014.

SÁNCHEZ CUADRADO, Adolfo. Aprendizaje formal de ele mediante actividades cooperativas de traducción pedagógica con atención a la forma. Tese de doutorado, 2016. Disponível em: <http://hdl. handle.net/10481/41765>> 
REVISTA X, Curitiba, volume 14, n.2, p.199-217,2019.

SCHJOLDAGER, Alan. Are 12 learners more prone to err when they translate. Translation in undergraduate degree programmes. Philadelphia: John Benjamins Publishing Company, 2004, p. 127-152.

SNELL-HORNBY, Mary. Translation as a means of integrating language teaching and linguistics. Translation in foreign language teaching and testing. Germany: Gunter Narr Verlag Tübingen, 1985, p. 21-28.

SNELLINGS, Patrick, VAN GELDEREN, Amos e DE GLOPPER, Kees. Lexical retrieval: An aspect of fluent second-language production that can be enhanced. Language Learning, no.52, 2002, p. 723-754.

SØRENSEN, Knud. Translation as a unifying discipline. Translation. A means to an end Aarhus: Aarhus University Press, 1990, p. 57-68.

TITFORD, Carl. Translation - a post-communicative activity for advanced learners. Translation in foreign language teaching and testing Germany: Gunter Narr Verlag Tübingen, 1995, p. 73-86.

VAEZI, Shanin. e MIRZAEI, Marius. The effect of using translation from 11 to 12 as a teaching technique on the improvement of efl learners' linguistic accuracy-focus on form. Humanising Language Teaching, no. 9, 2007. 
REVISTA X, Curitiba, volume 14, n.2, p.199-217, 2019.

\section{ANEXO A}

Este é o fragmento de um folheto para divulgar a Universidade de Houston. A tradução está dirigida a estudantes da Universidade de Cádiz que estejam interessados em estudar um ano da sua licenciatura nos Estados Unidos. Os folhetos serão distribuídos durante encontros informativos na Universidade de Cádiz.

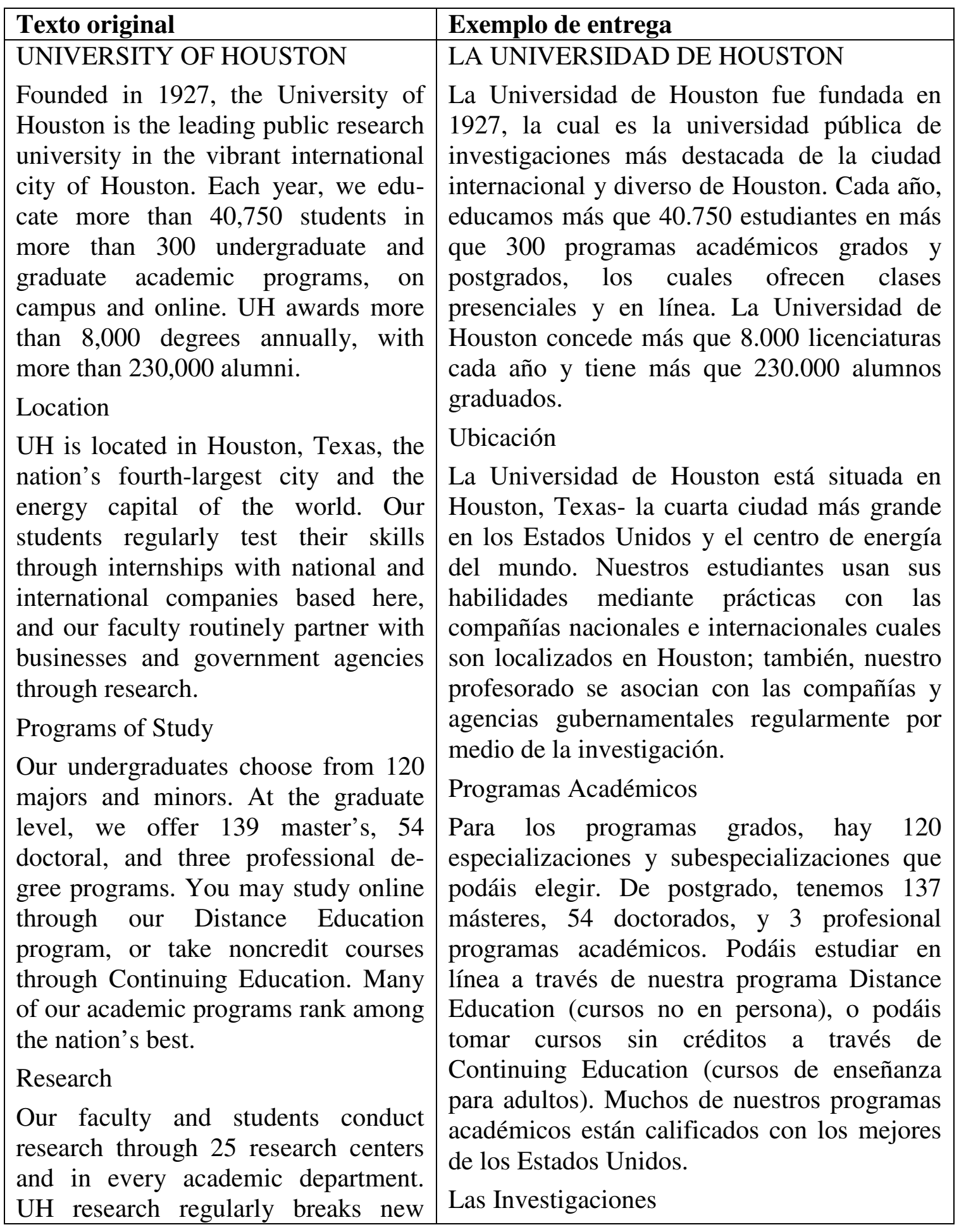


REVISTA X, Curitiba, volume 14, n.2, p.199-217,2019.

ground and opens doors to new ways of understanding the world.

Nuestro profesorado y los estudiantes hacen
investigaciones con 25 centros de
investigaciones y en cada facultad académica.
Los investigaciones de la Universidad de
Houston son revolucionarios así abren nuevos
caminos para entender el mundo de maneras
nuevas y diferentes.

Comentário de tradução:

A função do texto meta é divulgar a Universidade de Houston. O público são os estudantes da Universidade de Cádiz (que estejam interessados em estudar um ano da sua licenciatura nos Estados Unidos). A modalidade é um folheto. Estará disponível nos encontros informativos na Universidade de Cádiz.

\section{Dificuldades e estratégias:}

As dificuldades que encontrei eram de terminologia escolar porque há diferenças nos termos de educação nos Estados Unidos e na Espanha, então tinha termos diferentes de cada um e eu tinha que procurar qual devia usar e como usam na Espanha. Além disso, as metáforas requerem mais tempo de busca, como dizer a mesma ideia em outra língua.

Para solucionar os problemas que encontrei, usei o SpanishDict, WordReference e ReversoContext, sites que me ajudam a encontrar definições, frases, sinônimos e exemplos de palavras e também mostram seu uso em contexto real. Acho que usei todas as técnicas de tradução sem me dar conta, mas especificamente usei:

- Tradução literal: "Each year, we educate more than 40,750 students" > "Cada año, educamos más que 40.750 estudiantes";

- Transposição: "is the leading public research university" > "es la universidad pública de investigaciones más destacada";

- Modulação: "breaks new ground" > "abren nuevos caminos";

- Adaptação: "undergraduate programs" > "programas académicos grados";

- Compensação: "new ways of understanding the world" > "entender el mundo de maneras nuevas y diferentes";

- Explicitação: "Distance Education" > "Distance Education (cursos no en persona)"

\section{ANEXO B}

\section{Pré-teste e pós-teste}

NEW IPAD AIR

A new iPad Air appears to be on the way. According to some sources, Apple is likely to introduce an updated model of the Air during an event in mid-March, marking the line's first change in over a year. The Air 2 was released in October 2014, but Apple chose not to introduce an Air 3 this past fall, when it announced the iPad Pro and iPad Mini 4. There aren't many details on what to expect from the new iPad model, but the "9to5Mac" site points to earlier rumors saying the new Air may get additional speakers and a flash for its rear camera. Most notably, the site says that Apple has been testing a version of the Air that works with the Apple Pencil. It's not entirely surprising that Apple would want to bring that feature from the Pro to the standard Air, but it would be surprising to see that happen after just a few months. 